cancer in women under the age of 50, early onset prostate cancer in families with male breast cancer, and most ovarian cancers.

Original article Lorenzo Bermejo J and Hemminki K (2004) Risk of cancer at sites other than the breast in Swedish families eligible for $B R C A 1$ or $B R C A 2$ mutation testing. Ann Onc 15: 1834-1841

\section{TNF-a blockade in metastatic breast cancer}

Etanercept, originally developed for the treatment of rheumatoid arthritis, is a recombinant human soluble form of the p75 tumor necrosis factor- $\alpha$ (TNF- $\alpha$ ) receptor, which inhibits binding of TNF- $a$ to its cell surface receptor. The drug is also being investigated for its potential antitumor effect and results of a phase II study in metastatic breast cancer have recently been published.

Authors Madhusudan et al. carried out an open-label study in 16 women with advanced disease, in whom conventional therapy had failed. The patients received $25 \mathrm{mg}$ doses of etanercept twice weekly by subcutaneous injection, for a median duration of 8.1 weeks.

The drug was well-tolerated; the most common adverse events were fatigue and injection site reactions such as rash and swelling. A significant increase in plasma levels of TNF- $\alpha$ indicated biological response to therapy in all patients. Additionally, a cytokine release assay showed decreased levels of interleukin-6 and Human monocyte chemoattractant protein-1 in peripheral blood samples in response to phytohemagglutinin, suggesting that downstream TNF- $\alpha$-associated pathways were affected by the drug. Although no partial or complete responses were seen at this dose level, one patient achieved temporary disease stabilization after 12 weeks' therapy.

In summary, the study demonstrates the safety and biological activity of TNF-a blockade with etanercept in patients with metastatic breast cancer. Further studies will focus on different dosing schedules and combination with other therapies.

Original article Madhusudan S et al. (2004) A phase II study of Etanercept (Enbrel), a tumor necrosis factor a inhibitor in patients with metastatic breast cancer. Clin Cancer Res 10: 6528-6534

\section{Advances in thermal therapy for recurrent prostate cancer}

Interstitial microwave thermal therapy has been investigated as a treatment for certain solid tumors. Sherar et al. have applied this technology to the field of prostate cancer and have recently reported on their experience.

Thermal therapy is based on the selective coagulation of the tumor tissue; protection of the surrounding, normal tissue is crucial. Interstitial microwave therapy allows relatively precise control of the zone of thermal damage. Indeed, treatment simulations using phantom materials showed that the prostate can be targeted whilst the urethra is protected using a cooling mechanism. A Phase I/II trial examined the safety and efficacy of interstitial microwave therapy as an alternative to prostatectomy or hormone therapy in 41 patients with prostate cancer. All patients had recurrent disease following external beam radiation therapy. Disease-free survival was similar to that found using cryotherapy but, importantly, complication rates were lower.

The authors explain that the development of this technology will focus on the prediction of changes in tissue properties and blood flow during the heating process. It will also be important to determine the optimal level of protection of the normal tissue and to reduce cost and risk. Noting that the treatment might be appropriate for other patient groups, including those who have failed primary brachytherapy, Sherar et al. call for larger, multicenter trials in this area.

Original article Sherar MD et al. (2004) Interstitial microwave thermal therapy and its application to the treatment of recurrent prostate cancer. Int $J$ Hyperthermia 20: $757-768$

\section{Breast cancer risk in survivors of childhood cancer}

A new study by Kenney et al. has examined the risk of developing breast cancer in women who survived cancer as children. The results show an increased risk in those who survived childhood sarcomas or who received chest radiotherapy.

This retrospective study was based on data from the ongoing Childhood Cancer Survivor 